\title{
CEPC partial double ring scheme and crab-waist parameters
}

\author{
Dou Wang ${ }^{1}$ \\ IHEP \\ 19B, Yu Quan Road, Beijing, China \\ E-mail: wangd930ihep.ac.cn
}

Jie Gao, Feng Su, Ming Xiao, Yuan Zhang, Yiwei Wang, Bai Sha, Huiping Geng, Jiyuan Zhai, Tianjian Bian, Na Wang, Xiaohao Cui, Yuanyuan Guo, Qing Qin IHEP

19B, Yu Quan Road, Beijing, China

In order to avoid the pretzel orbit, CEPC is proposed to use partial double ring scheme in CDR. Based on crab waist scheme, we hope to either increase the luminosity with same beam power as Pre-CDR, or reduce the beam power while keeping the same luminosity in Pre-CDR. FFS with crab sextupoles has been developed and the arc lattice was redesigned to acheive the lower emittance for crab waist scheme.

Work Supported by National Foundation of Natural Sciences (11575218 and 11505198) and Key

Laboratory of Particle Acceleration Physics \& Technology in CAS.

38th International Conference on High Energy Physics

3-10 August 2016

Chicago, USA

\section{${ }^{1}$ Speaker}

(C) Copyright owned by the author(s) under the terms of the Creative Commons

Attribution-NonCommercial-NoDerivatives 4.0 International License (CC BY-NC-ND 4.0). 


\section{Introduction}

CEPC is a ring with a circumference of $54 \mathrm{~km}$ to house an electron - positron collider in phase-I and be upgraded to a super proton-proton Collider (SPPC) in phase-II. The designed beam energy for CEPC is $120 \mathrm{GeV}$, aims for Higgs study. Meanwhile CEPC should be compatible with $\mathrm{Z}$ study. The main constraint in the design is the beam lifetime due to beamstruhlung and the synchrotron radiation power, which should be limited to $50 \mathrm{MW}$ per beam, in order to control the total AC power of the whole machine. The target luminosity is $\sim 2 * 10^{34} \mathrm{~cm}^{-2} \mathrm{~s}^{-1}$ for Higgs and $\sim 1 * 10^{34} \mathrm{~cm}^{-2} \mathrm{~s}^{-1}$ for $\mathrm{Z}$.

After Pre-CDR, we developed a new idea called partial double ring scheme showed in Fig.1. Therefore, a pretzel orbit is not needed. With partial double ring scheme, we can consider crab waist on CEPC. The most important advantage of crab waist is that the beam-beam limit can be increased greatly.

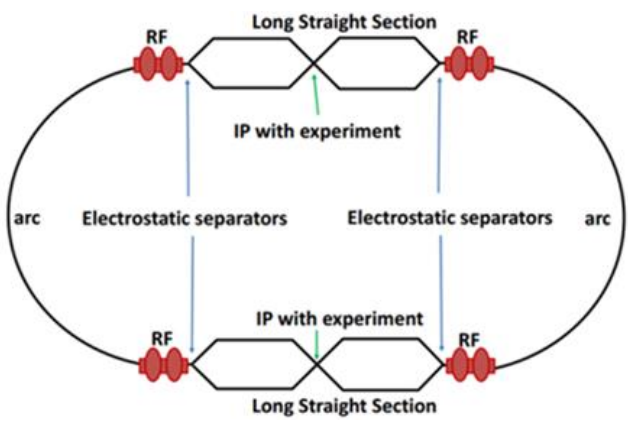

Fig. 1. CEPC partial double ring scheme.

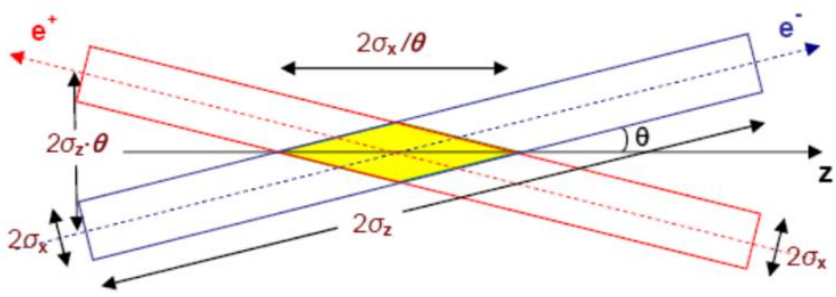

Fig. 2. Sketch of collision with crab waist.

\section{2. $\quad$ CEPC Parameter Choice for Partial Double Ring}

A general method of how to make an consistant machine parameter design of CEPC with crab waist by using analytical expression of maximum beam-beam tune shift and beamstrahlung beam lifetime started from given IP vertical beta, beam power and other technical limitations has been developed. Using this method, we get a set of new designs of CEPC overall parameters for $54 \mathrm{~km}, 88 \mathrm{~km}$ and $100 \mathrm{~km}$ circumference. $54 \mathrm{~km}$ CEPC parameter design was shown in table 1.

Table 1. CEPC crab waist parameters

\begin{tabular}{llllll}
\hline & Pre-CDR & H-high lumi. & H-low power & W & Z \\
\hline Number of IPs & 2 & 2 & 2 & 2 & 2 \\
Energy $(\mathrm{GeV})$ & 120 & 120 & 120 & 80 & 45.5 \\
Circumference $(\mathrm{km})$ & 54 & 54 & 54 & 54 & 54 \\
SR loss/turn $(\mathrm{GeV})$ & 3.1 & 2.96 & 2.96 & 0.59 & 0.062 \\
Half crossing angle (mrad) & 0 & 15 & 15 & 15 & 15 \\
Piwinski angle & 0 & 2.5 & 2.6 & 5 & 7.6 \\
Ne/bunch $\left(10^{11}\right)$ & 3.79 & 2.85 & 2.67 & 0.74 & 0.46 \\
Bunch number & 50 & 67 & 44 & 400 & 1100 \\
Beam current $(\mathrm{mA})$ & 16.6 & 16.9 & 10.5 & 26.2 & 45.4
\end{tabular}




\begin{tabular}{|c|c|c|c|c|c|}
\hline SR power /beam (MW) & 51.7 & 50 & 31.2 & 15.6 & 2.8 \\
\hline Bending radius $(\mathrm{km})$ & 6.1 & 6.2 & 6.2 & 6.1 & 6.1 \\
\hline Momentum compaction $\left(10^{-5}\right)$ & 3.4 & 2.5 & 2.2 & 2.4 & 3.5 \\
\hline$\beta_{I P \mathrm{x} / \mathrm{y}}(\mathrm{m})$ & $0.8 / 0.0012$ & $0.25 / 0.00136$ & $0.268 / 0.00124$ & $0.1 / 0.001$ & $0.1 / 0.001$ \\
\hline Emittance $\mathrm{x} / \mathrm{y}(\mathrm{nm})$ & $6.12 / 0.018$ & $2.45 / 0.0074$ & $2.06 / 0.0062$ & $1.02 / 0.003$ & $0.62 / 0.0028$ \\
\hline Transverse $\sigma_{I P}(\mathrm{um})$ & $69.97 / 0.15$ & $24.8 / 0.1$ & $23.5 / 0.088$ & $10.1 / 0.056$ & $7.9 / 0.053$ \\
\hline$\xi_{x} / \mathrm{IP}$ & 0.118 & 0.03 & 0.032 & 0.008 & 0.006 \\
\hline$\xi_{y} / \mathrm{IP}$ & 0.083 & 0.11 & 0.11 & 0.074 & 0.073 \\
\hline$V_{R F}(\mathrm{GV})$ & 6.87 & 3.62 & 3.53 & 0.81 & 0.12 \\
\hline$f_{R F}(\mathrm{MHz})$ & 650 & 650 & 650 & 650 & 650 \\
\hline Nature $\sigma_{z}(\mathrm{~mm})$ & 2.14 & 3.1 & 3.0 & 3.25 & 3.9 \\
\hline Total $\sigma_{z}(\mathrm{~mm})$ & 2.65 & 4.1 & 4.0 & 3.35 & 4.0 \\
\hline HOM power/cavity $(\mathrm{kw})$ & 3.6 & 2.2 & 1.3 & 0.99 & 0.99 \\
\hline Energy spread (\%) & 0.13 & 0.13 & 0.13 & 0.09 & 0.05 \\
\hline Energy acceptance $(\%)$ & 2 & 2 & 2 & & \\
\hline Energy acceptance by RF (\%) & 6 & 2.2 & 2.1 & 1.7 & 1.1 \\
\hline$n_{\gamma}$ & 0.23 & 0.47 & 0.47 & 0.3 & 0.24 \\
\hline $\begin{array}{l}\text { Life time due to beamstrah- } \\
\text { lung_cal (minute) }\end{array}$ & 47 & 36 & 32 & & \\
\hline F (hour glass) & 0.68 & 0.82 & 0.81 & 0.92 & 0.95 \\
\hline$L_{\max } / \operatorname{IP}\left(10^{34} \mathrm{~cm}^{-2} \mathrm{~s}^{-1}\right)$ & 2.04 & 2.96 & 2.01 & 3.09 & 3.09 \\
\hline
\end{tabular}

\section{FFS design and Crab sextupole parameter}

The lattice design of FFS (betax $=0.25 \mathrm{~m}$, betay $=0.00136 \mathrm{~m}$ ) for CEPC partial double ring is shown in Fig. 3. The $L^{*}$ is $1.5 \mathrm{~m}$ and the strength of first quadrupole (twin aperture) is $200 \mathrm{~T} / \mathrm{m}$. The critical energy of the whole system is under $190 \mathrm{keV}$.

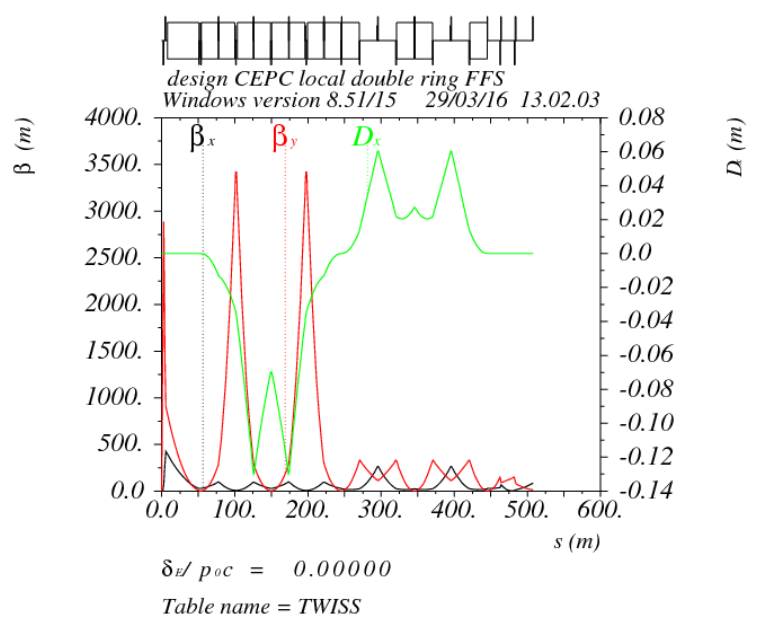

Fig. 3. FFS optics for CEPC partial double ring.

The crab sextupole should be placed on both sides of the IP in phase with the IP in the horizontal plane and at $\pi / 2$ in the vertical one. As Oide said, the second FFS sextupoles of the CCS-Y section can work as the crab sextupoles, if their strengths and phases to the IP are properly chosen.

The crab sextupole strength should satisfy the following condition depending on the crossing angle and the beta functions at the IP and the sextupole locations:

$$
\begin{aligned}
K L & =\frac{1}{2 \theta} \frac{1}{\beta_{y}^{*} \beta_{y}} \sqrt{\frac{\beta_{x}^{*}}{\beta_{x}}}=1.27 m^{-2} \\
K_{2} & =4.2 m^{-3}
\end{aligned}
$$




\section{Low emittance arc}

We tried to get smaller emittance as the parameter table 1. By reducing the FODO length from $47 \mathrm{~m}$ to $37 \mathrm{~m}$ and increase the phase of FODO cell, we got $2.3 \mathrm{~nm}$ emittance.
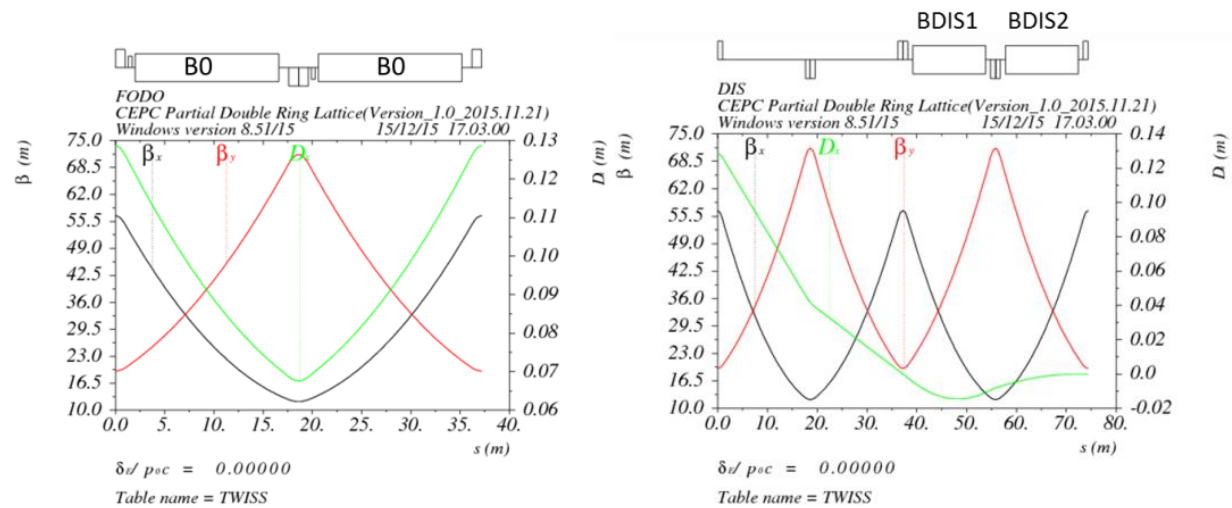

Fig. 4. Low emittance arc with $90^{\circ} / 60^{\circ}$ phase advance (left: FODO, right: dispersion supressor)
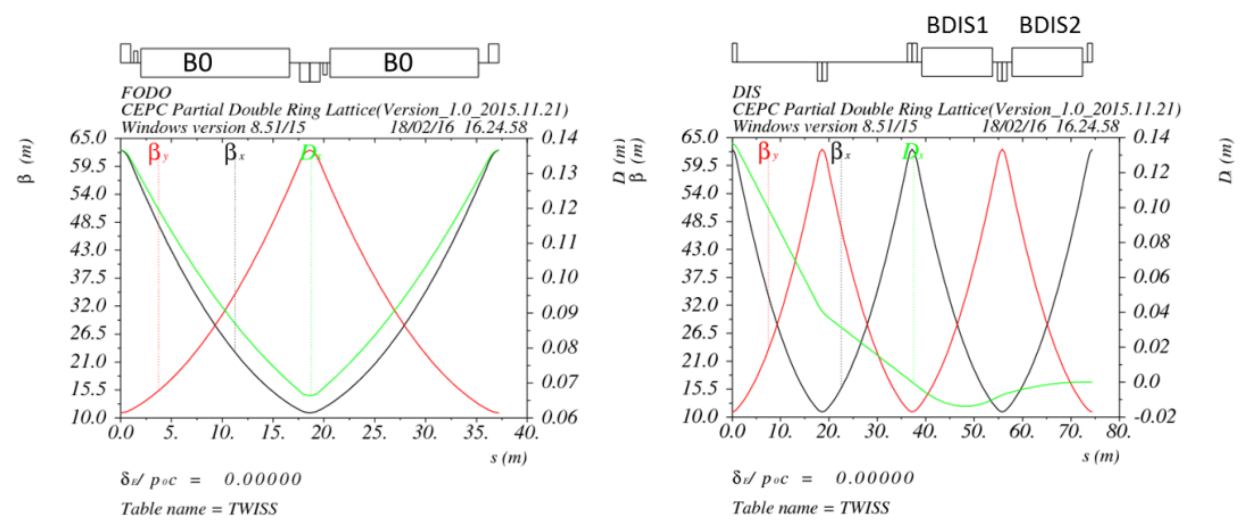

Fig. 5. Low emittance arc with $90^{\circ} / 90^{\circ}$ phase advance (left: FODO, right: dispersion supressor)

\section{Dynamic aperture}

With FFS, partial double ring, low emittance arc $\left(90^{\circ} / 90^{\circ}\right)$ and the bypasses of pp detectors together, we got a satisfying dynamic aperture for on momentum particle. So far, we have used 192 groups of sextupoles in the arc. The further optimization of DA bandwidth is underway.

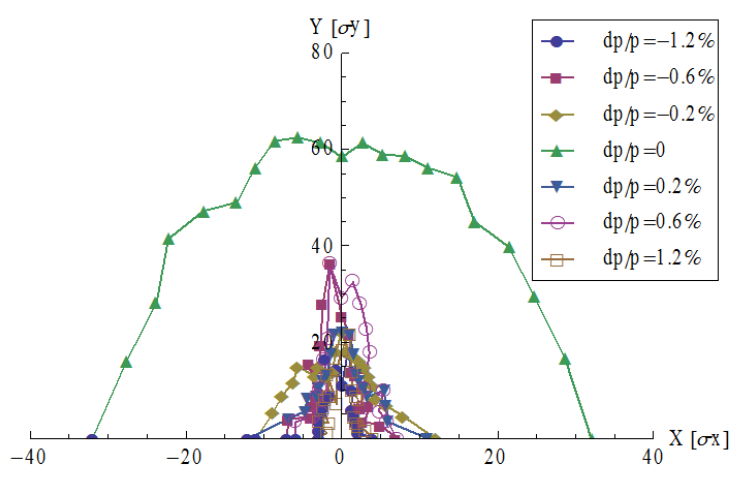

Fig. 6. FFS optics for CEPC partial double ring.

\section{Conclusion}


In this paper, a general method of how to make an consistant machine parameter design of CEPC with crab waist by using analytical expression of maximum beam-beam tune shift and beamstrahlung beam lifetime started from given IP vertical beta, beam power and other technical limitations was developed. Based on this method, a set of optimized parameter designs for $54 \mathrm{~km} \mathrm{CEPC} \mathrm{with} \mathrm{partial} \mathrm{double} \mathrm{ring} \mathrm{scheme} \mathrm{were} \mathrm{proposed.} \mathrm{Crossing} \mathrm{angle} \mathrm{was} \mathrm{fixed} \mathrm{at}$ $30 \mathrm{mrad}$ both for Higgs $\mathrm{W}$ and $\mathrm{Z}$. Thanks to the beam-beam limit enhancement effect of crab waist, we can either get higher luminosity with same beam power as Pre-CDR, or reduce the beam power by $40 \%$ keeping the same luminosity in Pre-CDR. Both proposals should improve the performance of CEPC. For "H-high lumi" mode, the HOM power per cavity can not be reduced under $1 \mathrm{kw}$. The minimum value is about $2 \mathrm{kw}$. In addition, the optics of FFS has been designed and the strength of crab sextupole has been estimated, and so far this kind of sextupole is available.

Based on partial double ring scheme, we get a set of $\mathrm{Z}$ parameter with $3.1 \times 10^{34} \mathrm{~cm}^{-2} \mathrm{~s}^{-1}$ luminosity using 1100 bunches.

Lower emittance arc with $2.3 \mathrm{~nm}$ emittance has been designed both for $90^{\circ} / 60^{\circ}$ case and $90^{\circ} / 90^{\circ}$ case. The dynamic aperture of the whole ring is good enough for on momentum particle while the off momentum DA is still need to been optimized.

\section{Acknowledgements}

The authors thank Professors Yunhai Cai, Michael Koratzinos, Katsunobu Oide and Valery Telnov for their helpful discussions.

\section{References}

[1] The CEPC-SPPC Study Group, CEPC-SPPC preliminary Conceptual Design Report, Volume IIAcceleraor, IHEP-CEPC-DR-2015-01, IHEP-AC-2015-01, March 2015.

[2] D. Wang, J. Gao et al., Optimization parameter design of a circular $\mathrm{e}^{+} \mathrm{e}^{-}$Higgs factory, Chinese Physics C, Vol. 37, No. 9 (2013) 097003.

[3] M. xiao, J. Gao, D. Wang, F. Su, Y. Wang, S. Bai, T. Bian, Study of CEPC performance with different collision energies and geometric layouts, Chinese Physics C, 2016, accepted.

[4] Jie Gao, 关于 CEPC 采用亚毫米 $\beta y$ 带角度对撞以减少辐射功率并保证对撞亮度的 Lattice 优化 设计建议, IHEP-AC-LC-Note2013-012, in Chinese.

[5] Mike Koratzinos, private communication.

[6] J. Gao, emittance growth and beam lifetime limitations due to beam-beam effects in e+e- storage rings, Nucl. Instr. and methods A533 (2004) p. 270-274.

[7] V. Telnov, Restriction on the energy and luminosity of e+e- storage rings due to beamstrahlung, arXiv:1203.6563v, 29 March 2012.

[8] V. Telnov, Limitation on the luminosity of e+e- storage rings due to beamstrahlung, HF2012, November 15, 2012.

[9] V. Telnov, Issues with current designs for e+e- and gammagamma colliders, PoS Photon2013 (2013) 070. https://inspirehep.net/record/1298149/files/Photon\%202013 070.pdf 